\title{
ARECA-COPT: Pesticide Sprayer Quad Copter
}

\author{
Shraddha ${ }^{1}$, Shwetha Rathnakar Shetty ${ }^{2}$, Varsha $K^{3}$, Veenashree ${ }^{4}$, Sachin Bhat ${ }^{5}$ \\ Department of Electronics \& Communication Engineering, Shri Madhwa Vadiraja Institute of Technology \& \\ Management, Bantakal, India ${ }^{1,2,3,4,5}$
}

\begin{abstract}
In the recent year UAV( Unmanned Aerial Vehicle) having quadcopter helicopter i.e., quadcopter configuration has been receiving increasing attention amongst the global researchers due its wide range of applications such as surveillance in military, civilian, and disaster management application. Here we try to implement its application to agriculture field. In agricultural fields, the use of pesticides is necessary for better crop yields. The use of aircrafts and drones has become common for carrying out this operation mainly because of its speed and effectiveness in the spraying operation. The problem with manual spraying is the lack labour. Also manual spraying cause direct health problem. The WHO (World Health Organization) estimates there are more than 1 millionpesticide cases in every year. In that more than one lakh deaths in each year, especially indeveloping countries due to the pesticides sprayed by human being.This project is to mainly overcome the ill-effects of pesticides on human beings(manual pesticide sprayers) and also to cover larger areas while spraying pesticides in a short span of time when compared to a manual sprayer.
\end{abstract}

Keywords: Quad copter, BLDC motor, flight controller, RF transmitter and receiver.

\section{INTRODUCTION}

India is a developing country. It has a rich land source which is the best suited for agriculture. Rural people, who constitute nearly 70 per cent of population, are dependent on agriculture as a main occupation and have a significant impact on the national income. Currently, agriculture field is facing problems due to lack of human labour as it involves more physical work and expenditure. Hence field workers are migrating from farm to other fields in industry which offer more stable and more profitable jobs. In this scenario, it becomes necessary to introduce and develop agricultural automation and technologies to increase agricultural productivity by providing agricultural implements which are most sophisticated and can be procured by the farmers at feasible rates and with ease of access. Present day in agriculture the sprayers play an important role in spraying pesticides. Although sprayer specification varies with motorized, hand operated and in helicopter. The current idea on sprayer in our project is to utilize effectively and thus reduce the health problems caused by manual spraying.

\section{LITERATURE REVIEW}

Earlier the foot walking pesticide spraying machine is fitted on the foot activated by kinetic force being applied on it, when we stamp our foot on the ground, pushing the assembly to pump air into the tank, the power is very less but sufficient enough to use the pressure for pesticide spraying. Since we are using two numbers of pumps which pumps the air into the tank and has two number of jet sprayers, we can cover two parallel rows simultaneously [1]. Later there was a scheme of an intelligent control and navigation system of an indoor quad copter. Innovations are focused on achieving the same control and navigation systems with low cost components. In addition, the independency of the quad-copter is increased as there is no high requirement for the environment and no preinstalled equipment required to perform the navigation. Traditional PID controller, Raspberry Pi on-board flight computer and electronic speed controller was developed to provide the basic platform for the quad-copter [2]. In GIS based Fire Rescue System for Industries the adoptability of UAV's (Quad copter) in acquiring and communicating information in hazardous environment. The airborne vehicles are enduring, cost effective and also provide a reliable platform for surveying. Consequently, small airborne vehicles, here Quad copter, equipped with sophisticated components can capture and transmit the image or video to the Data Base. They are sent for processing and made accessible to the Geographical Information System (GIS), where further analysis is done for the proceedings of the rescue operation. This application finds a great utility predominantly in industries and military [3]. For Surveillance thermal camera was used and determines the land mine by the help of surveillance drone. Using a conventional land robot for detection may result in blowing up the mine, which might prove to be an expensive affair and hence by using a quadcopter which has a mine detector mounted on it as it can be controlled at a distance of a kilometre remotely from another location. This utilized two different modes of detection which are an IR camera and a metal detector. These are extensively used in aiding this whole operation. This model also gives provision for further improvement both in terms of conserving time and 
improving the range of transmission system [4]. Thus, from [2], [3], [4] the idea of utilization of quadcopter for pesticide spraying for agricultural purposes was determined. For pesticide spraying, it was focused on quad copter which had a capacity to carry the pesticides which could be sprayed. The height of spraying is around 6-7 feet. The area covered is 10 feet by 10 feet. The flight time of the quad copter is very less [5]. In Designing and Development of a Quad Copter Using CAD, the developing of a quad copter configuration MFV which had a capability of carrying out surveillance from $20 \mathrm{~m}$ height. Take-off and landing weights (masses) were $0.346 \mathrm{~kg}$. Its primary application was to reduce hazardous or toxic environment which was unsuitable for humans [6].

\section{III.METHODOLOGY}

Fig 3.1 shows the proposed block diagram of the quad copter. It consists of the flight controller, radio receiver, power supply, electronic speed controllers (ESC), brushless DC motors (BLDC).Controlling of the quad copter is done through the radio transmitter.The flight controller is the main part of this vehicle. This flight controller controlsall the operation commanded by us. Thefour rotors to create differential thrust andthe quadcopter hover and moveaccordance with the speed of those rotors. There are two types of configuration inquadcopter construction. First one is Plus(+) configuration and another one is $\operatorname{Cross}(\mathrm{X})$ configuration. In this project we used $\mathrm{X}(\mathrm{Cross})$ configuration. The brushless motors are multi-phased, normally 3 phases, so direct supply of DC power will not turn the motors on.BLDC electric motor also known as electronically commutated motors.The ESC's are generating three high frequency signals with different but controllable phases continually to keep the motor turning. The ESC is also able to source a lot of current as the motors can draw a lot of power.

Another part of our project is sprayer. Fig 3.2 shows the block diagram of sprayer. It consists of a pump, nozzle, power supply, and switch. The pump of the sprayer module is controlled by RF transmitter.Servo switch used for actuation of the pump depending on the control signal given the RF transmitter. Wherever need to activate the sprayer, just comment byremote RF transmitter.

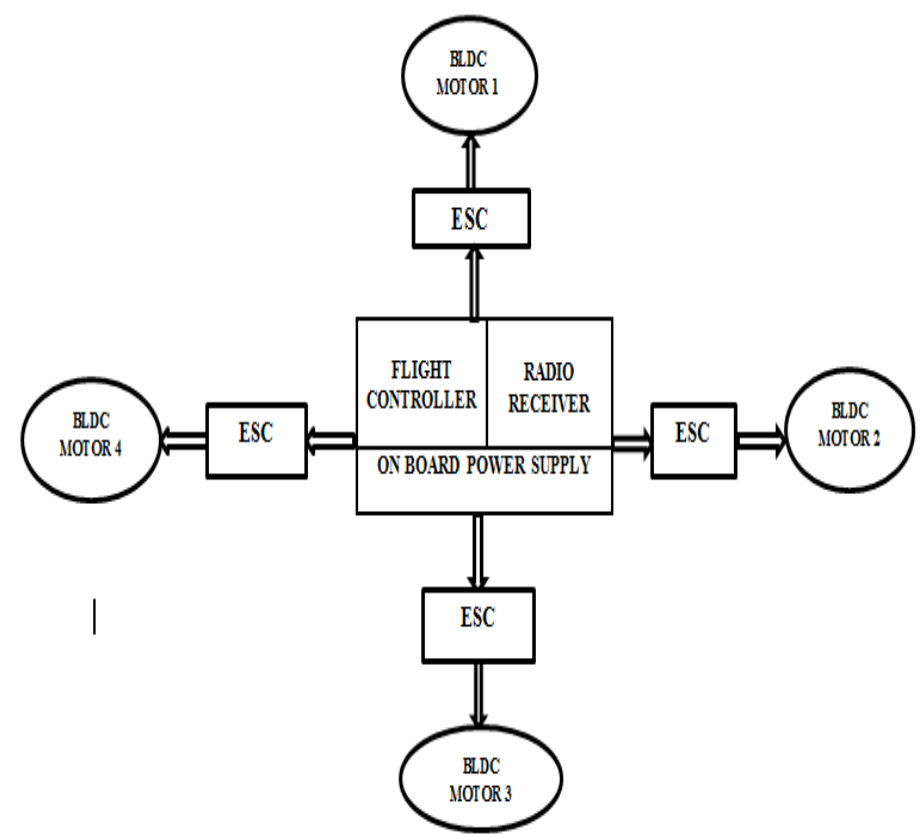

Fig. 3.1 Block diagram of Quad copter

NOZZLE:

A nozzle is a device designed to control the direction or characteristics of a fluid flow (especially to increase velocity) as it exits (or enters) an enclosed chamber or pipe.

A nozzle is often a pipe or tube of varying cross sectional area, and it can be used to direct or modify the flow of a fluid (liquid or gas). Nozzles are frequently used to control the rate of flow, speed, direction, mass, shape, and/or the pressure of the stream that emerges from them. In a nozzle, the velocity of fluid increases at the expense of its pressure energy.Here we used a simple nozzle usually used for spraying water or other liquids. 


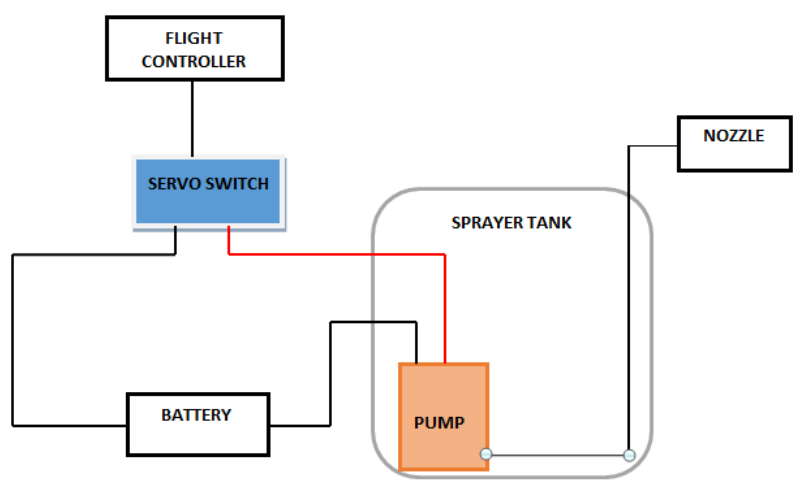

Fig. 3.2 Block diagram of spraying system

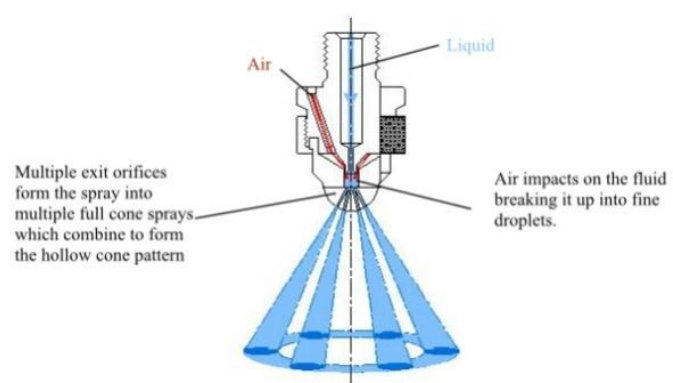

Fig. 3.3 Block diagram of nozzle

\section{IV.HARDWARE DESCRIPTION}

FRAME

Every quadcopter or other multirotor aircraft needs a frame to house all the other components. . Its only purpose is to attach the motors to each other, and to support the electronics. The frame needs to be as stiff as possible to provide good handling characteristics. These two goals are contradictory to each other, as the stiffer we make a frame, the heavier it will be. Here we have used balsa wood for the quadcopter frame. . Balsa wood has been used in RC Aircraft forever, and for good reason. It is inexpensive, extremely light, and fairly stiff and strong.

\section{MOTORS}

Brushless DC electric motor also known as electronically commutated motors are synchronous motors that are powered by a DC electric source via integrated inverter/switching power supply, which produces an AC electric signal to drive the motor. The brushless motors are multi-phased, normally 3 phases, so direct supply of DC power will not turn the motors ON. The motors have an obvious purpose: to spin the propellers. Motors are rated by KV rating, higher the KV rating the faster the motor spins at a constant voltage. In this project we have used DM2810-KV1200 brushless motor which gives a maximum efficiency of $84 \%$.

Table No. 4.1 Technical data of BLDC motor

\begin{tabular}{|l|l|}
\hline \multicolumn{2}{|c|}{ Technical Data } \\
\hline KV & 1200 \\
\hline Weight(g) & 91 \\
\hline Motor Dimension(W*L/mm) & $39 * 27$ \\
\hline Shaft Size(mm) & 5 \\
\hline Max Efficiency & $84 \%$ \\
\hline Max current(A) & 40 \\
\hline Max Thrust(g) & 1850 \\
\hline Max Power & $500 \mathrm{~W} / 4 \mathrm{~S}$ \\
\hline
\end{tabular}




\section{FLIGHT CONTROLLER}

A flight controller is the brain of the quadcopter. It is basically a circuit board that takes sensors data and user commands, and controls the motors in order to keep the quadcopter in the air. It houses the sensors such as gyroscopes and accelerometers that determine how fast each of the quadcopter's motors spin. CC3D is a flight controller that is developed by Open Pilot project, it stands for Copter Control 3D.Currently supporting two platforms, the CC3D and the Revolution (Revo). CC3D features a powerful STM32 32-bit microcontroller running at 90MIP's with 128KB Flash and 20KB RAM. It has 3- axis high performance gyros and 3 axis high performance MEMs accelerometer and 4Mbits on-board EEPROM for configuration storage. Flexi ports give copter control the flexibility to use either I2C and a single serial port or dual serial ports, according to connectivity needs. The included RC harness allows the user to connect ant standard PWM receiver. A standard mini-USB cable is used to load the latest firmware and configure everything for the unique airframe.

\section{RF TRANSMITTER AND RECEIVER}

The radio transmitter and receiver allow you to control the quad copter. The avionic RCB6i is one of best transmitter in the market for beginners. It is $2.4 \mathrm{GHz}$ FHSS technology provides a highly secure connection, optimum responsiveness, increased range and the ability to fly more safely. This radio allows the user to interchange from mode 1 to mode 2 with the use of slider at the back of the radio. It also provide unique range test feature. It provides LCD to enable the user to check their setting in any surrounding. It contains digital programmable transmitter with 6- channel able to effectively control model airplanes.

\section{LiPo BATTERY}

Initially there was a use of Nickel Cadmium (NiCad) and Nickel Metal hybrid (NiMH) were first used, but lithium based batteries have now taken over. The reason for the popularity of lithium battery is simple-really high energy density. Lithium batteries have enough stored energy to let quad copter to fly for at least a useful length of time. The current rating of a battery refers the total energy available from the battery at its rated voltage. The rating is in $\mathrm{mAh}$ and is available current that can e provided by the battery for one continuous hour. We selected a battery of $11.1 \mathrm{v} 3 \mathrm{~s}$ 4000mah 30c LiPo battery which has heavy duty discharge leads to minimize resistance and sustain high current loads. Here $4000 \mathrm{mAh}$ means battery may be discharged at $1 \mathrm{Amp}$ for 1 hour. Here we used $11.1 \mathrm{v} 3 \mathrm{~S}$ which refers to three 3.7 volt LiPo cells in series producing a nominal 11.1v. A battery's C (capacity) rating is the maximum safe continuous discharge rate if battery. A batteries maximum acceptable discharge rate is calculated by multiplying its current rating by its $\mathrm{C}$ rating. Here we used $30 \mathrm{C}$ which refers that battery can be discharged at the same rate as its capacity.

\section{PROPELLERS}

Propellers come in a variety of diameters and pitches as well as materials such as plastic, reinforced plastic, carbon fiber and wood. Smaller propellers fewer than 8 inches are used for racing and acrobatics along with motors of high $\mathrm{kV}$ rating. While choosing the propeller we should decide its pitch. Pitch is defined as the travelling distance per a single revolution of the propeller. Lower pitch will generate more torque for lifting and the motors don't have to work as hard to carry heavier loads. As a result, a motor that doesn't have to work as hard will draw less current from the battery which results in increased flight time. Here the pitch selected is 4.7 which defines that the propeller is capable of 4.7 inches of travel per revolution. The propeller which we selected is $11 * 4.7$ where first number indicates the diameter and second number indicates the pitch. Computer-optimizes design gives these props a thinner profile and more bite with less noise. The propeller which is used here has light weight molded nylon construction reduces rotating mass for higher output and longer life. Reinforcing carbon fibers help maintain true, constant pitch at any rpm.

\section{RESULT AND DISCUSSION}

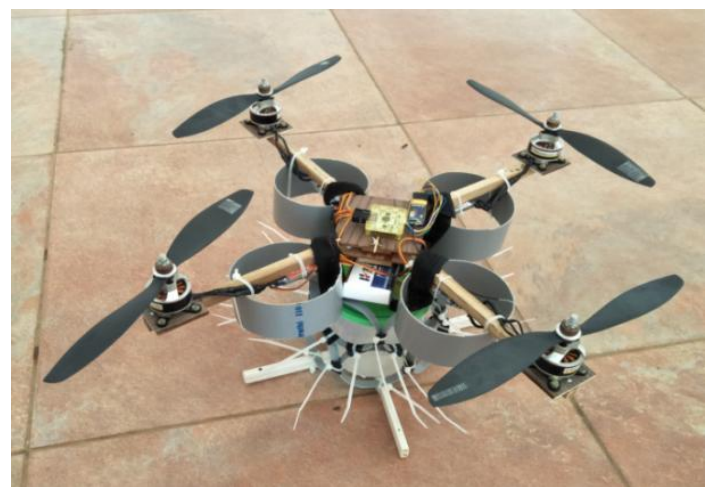




\section{International Journal of Innovative Research in Electrical, Electronics, Instrumentation and Control Engineering}

ISO 3297:2007 Certified

Vol. 5, Issue 5, May 2017
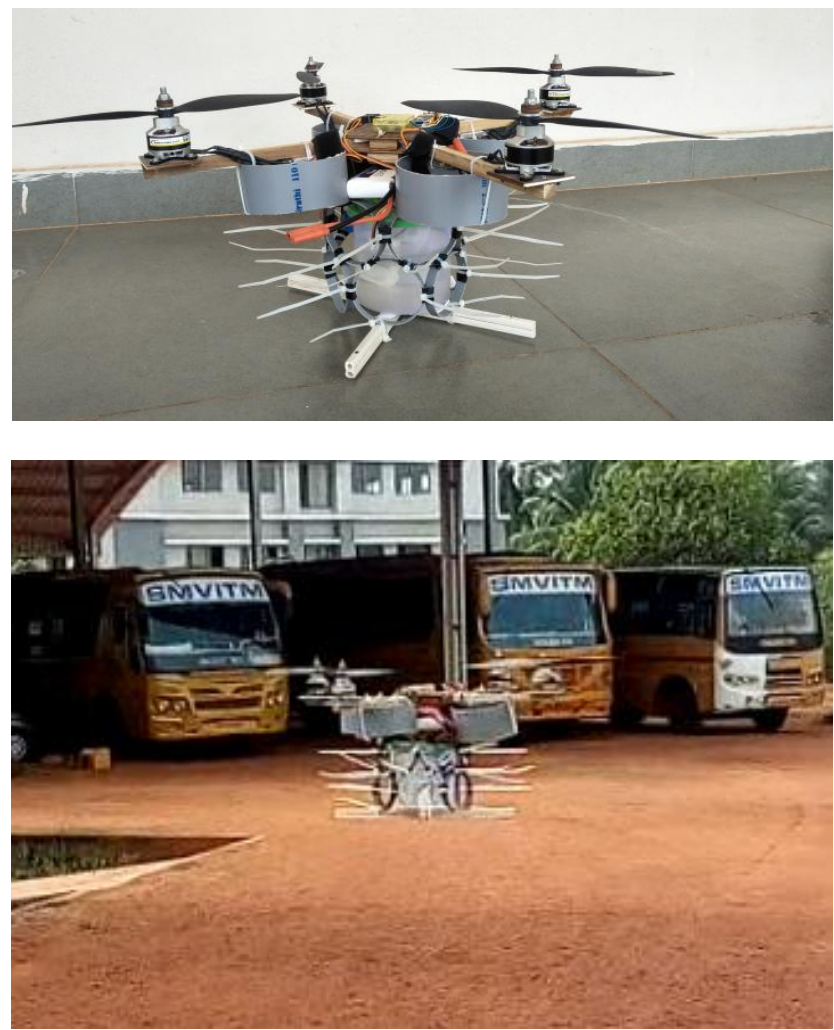

Fig. 5.1 Hardware Model of Proposed Method

The spraying time of pesticides is dependent on the quantity of pesticide to be sprayed. For example, for $1000 \mathrm{ml}$ of pesticides, spraying time is around 5 minutes. If we want to increase the quantity of pesticide to be sprayed, the weight lifting capacity of the quadcopter must be increased. This is done by choosing higher specification of BLDC i.e., more than $1000 \mathrm{rpm} / \mathrm{kV}$. The flight time of the quadcopter is 12 minutes. The proposed method can carry a payload of $1.5 \mathrm{~kg}$ and flight time with sprayer will be 5-6 min. To increase the flight time we need to choose higher specification for LiPo battery.The height of spraying is around nearly 15 feet.

\section{VI.CONCLUSION}

For a country like India where agriculture is the backbone, different development methods should be implemented. Since agriculture field is facing problems due to lack of human labor as it involves more physical work and expenditure, it becomes necessary to introduce and develop agricultural automation and technologies to increase agricultural productivity by providing agricultural implements which are most sophisticated and can be procured by the farmers at feasible rates and with ease of access. Furthermore, present day, in agriculture, the sprayers play an important role in spraying pesticides. Thus our project aimed in meeting problems in spraying pesticides to arecanut trees where farmers face problems like health issues, lack of labor etc. Our project provides a flight time of 6 minutes with a payload of $1.5 \mathrm{~kg}$. Thus it saves the time of farmer to do other useful work instead of searching for labors and also once he has such a system he need not invest extra money on labors.

\section{REFERENCES}

[1]S R Kulkarni, R V Nyamagoud, HareeshNaik, Mohan Futane, "Fabrication of Portable Foot Operated Agricultural Fertilizers and Pesticides Spraying Pump", International Journal of Engineering Research \& Technology (IJERT), ISSN: 2278-0181, Vol. 4, Issue-07, July-2015.

[2]YiwenLuo, MengJooEr, Li Ling Yong, Chiang JuChien, "Intelligent Control and Navigation of an Indoor Quad-copter", 13th International Conference on Control, Automation, Robotics \& Vision Marina Bay Sands, Singapore, 10-12th December 2014 (ICARCV 2014).

[3]P Vijay Kumar, AkhilChalla, Jammi Ashok, G. Lakshmi Narayanan, "GIS based Fire Rescue System for Industries using Quad copter -A Novel Approach", International conference on microwave, optical and communication engineering, December 18-20, 2015, IIT Bhubaneswar, India.

[4] Yuvaraj Ganesh, RamyaRaju, RajeshwariHegde, "Surveillance Drone for Landmine Detection", International Conference on Advanced Computing and Communications, 2015.

[5] MisbahRehman.Z, Kavya.B, Divya Mehta, PriyaRanjan Kumar, Prof. Sunil Kumar G.R, "Quad-copter for pesticide spraying", International Journal of Scientific \& Engineering Research, Vol. 7, Issue-5, May-2016, ISSN 2229-5518. 


\section{IJIREEICE \\ International Journal of Innovative Research in Electrical, Electronics, Instrumentation and Control Engineering \\ ISO 3297:2007 Certified \\ Vol. 5, Issue 5, May 2017}

[6] IrfanAnjumManarvi, Muhammad Aqib, Muhammad Ajmal, Muhammad Usman, SaqibKhurshid, UsmanSikandar, "Design and Development of a Quad Copter (UMAASK) Using CAD/CAM/CAE”, Department of Mechanical Engineering, HITEC University, Taxila Education City, Taxila, Pakistan, IEEE,2013.

\section{BIOGRAPHIES}

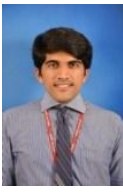

(Dr) SACHIN BHAT Assistant professor, ECE department, SMVITM, Bantakal.

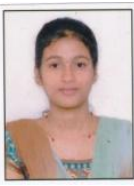

VEENASHREE $8^{\text {th }}$ sem, student ECE department, SMVITM, Bantakal.

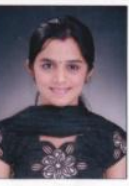

SHRADDHA BHAT $8^{\text {th }}$ sem, student, ECE department, SMVITM, Bantakal.

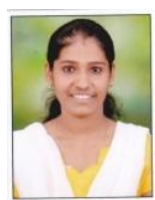

VARSHA K $8^{\text {th }}$ sem, student, ECE department, SMVITM, Bantakal.

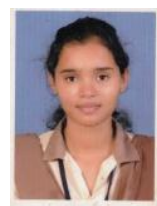

SHWETHA RATHNAKAR SHETTY $8^{\text {th }}$ sem, student, ECE department, SMVITM, Bantakal. 